TRANSACTIONS OF THE

AMERICAN MATHEMATICAL SOCIETY

Volume 186, December 1973

\title{
DIFFEOMORPHISMS HOMOTOPIC TO THE IDENTITY
}

\author{
BY
}

EDWARD C. TURNER(1)

ABSTRACT. In this paper, an inductive procedure for describing the group of isotopy classes of null homotopic diffeomorphisms of a manifold is developed -this process depends on the handlebody structure of the manifold. This group is also shown to be finitely generated and in many cases abelian.

0. Introduction. We present an inductive procedure for describing the group $D(M)$ of isotopy classes of diffeomorphisms of a simply connected manifold that yields particularly strong information about the subgroup $D^{\pi}(M)$ homotopic to 1 ( 1 = the identity). A "resolution" of $D^{\pi}(M)$ is obtained which relates the calculation of $D^{\pi}(M)$ to groups of framed knots and homotopy problems. (We use $D$ and $E$ to denote spaces of diffeomorphisms and embeddings and $D$ and $E$ to denote the sets of isotopy classes.) This resolution is applied in [T] to describe a family of manifolds with finite diffeomorphism groups. We also show that $D^{\pi}(M)$ is finitely generated (even if $\left.\pi_{1}(M) \neq 0\right)$ and in many cases $\left(\pi_{1}(M)=0\right.$ and $\operatorname{dim}(M)$ odd $)$ is abelian. The development is straightforward and requires only one deep result, the following corollary of a theorem of Browder [B].

Theorem. If $N^{n-k} \subset M^{n}, M$ is 2-connected, $N$ simply connected and $k \geq 3$, then if $d \in \mathfrak{D}^{\pi}(M), d \mid N$ is isotopic to the inclusion, modulo local knotting.

The procedure starts with a handlebody resolution of $M$ (which we assume to be minimal)

$$
D^{n}=M_{0} \subset M_{1} \subset \ldots \subset M_{l}=M,
$$

and the associated chain of subgroups

$$
\mathscr{D}\left(D^{n}\right)=\mathscr{D}\left(M_{0}\right) \subset \ldots \subset \mathcal{D}\left(M_{l}\right)=\mathscr{D}(M)
$$

Received by the editors November 11, 1971 and, in revised form, November 22, 1972, and May 10, 1973.

AMS (MOS) subject classifications (1970). Primary 57D50.

Key words and phrases. Diffeomorphism groups, isotopy, homotopy, surgery exact sequence.

(1) Partially supported by National Science Foundation Grant SD GU 3171. 
where $\mathcal{D}\left(M_{i}\right)$ is the group of diffeomorphisms of $M_{i}$ which are 1 on $\partial M_{i}$ and $\mathscr{D}\left(M_{i}\right) \subset \mathscr{D}\left(M_{i+1}\right)$ is the extension by 1 over the handle. In $\S 1$, we show that $\mathscr{D}\left(M_{i}\right)$ is fibered over an appropriate space of embeddings with $\mathscr{D}\left(M_{i-1}\right)$ as fiber. Taking components,

$$
\Gamma_{n+1}=\pi_{0}\left(D\left(M_{0}\right)\right) \rightarrow \cdots \rightarrow \pi_{0}\left(D\left(M_{l}\right)\right)=\pi_{0}(D(M))
$$

has cokernels which are sets of isotopy classes of embeddings. Unless strong restrictions are put on the manifold, these sets are very hard to calculate. However, if we make the requirement that everything be homotopic to 1 (indicated by a superscript $\pi$ ) the cokemels can be described in terms of framed knots, about which much is known, and homotopy terms. This description is presented in $\$ 2$. In $\$ 3$, we adopt a different viewpoint and prove that $D^{\pi}(M)$ is finitely generated and often abelian.

All manifolds will be assumed to be connected and simply connected and diffeomorphisms to be $C^{\infty}$. Unless stated to the contrary, all diffeomorphisms are the identity on the boundary. The author wishes to thank his colleagues, particularly Dennis Sullivan, for many helpful discussions, and the reviewer for his suggestions concerning $\$ 3$.

1. The fibrations. Let $N^{n}$ and $M^{m}$ be compact $C^{\infty}$ manifolds (possibly with boundary) and $N^{\prime}$ be a closed $(n-1)$-dimensional submanifold of $\partial N$. If $i$ : $N^{\prime} \rightarrow M$ is a specific embedding then

$$
\left.\mathcal{E}_{i}(N, M)=\text { embeddings } b: N \rightarrow M \mid b=i \text { on } N^{\prime} \text { and } b^{-1}(\partial M)=N^{\prime}\right\}
$$

with the $C^{k}$ topology, $2 \leq k \leq \infty$. $\mathcal{E}_{i}(N, M)$ is a Hilbert manifold if $2 \leq k<\infty$ and a Fréchet manifold if $k=\infty$-as the homotopy type of $\mathcal{E}_{i}(N, M)$ does not depend on $k$, and we are interested only in homotopy results, we will choose $k$ to suit the needs of the moment. Notice that for $k<\infty, \mathcal{E}_{i}(N, M)$ has a natural metric and so is paracompact. In the case that $N=M, N^{\prime}=\partial M$ and $i$ is the inclusion, $\xi_{i}(N, M)=\mathscr{D}(M)$, the diffeomorphisms of $M$ modulo $\partial M$.

Now suppose $\bar{i}: N \rightarrow M$ is a specific embedding in $\tilde{E}_{i}(N, M)$ and $R: D(M) \rightarrow$ $\xi_{i}(N, M)$ by restriction; i.e., $R(f)=f \circ \bar{i}$. Then

\section{Theorem 1.1: $R$ is a locally trivial fibration.}

Using the Hilbert (or Fréchet) manifold descriptions and fairly direct generalizations of techniques of Palais (see, e.g., [P]) the local product structure can be described in terms of vector field extensions. To show that it is a fibration, however, requires only the verification of what Hu calls the "polyhedral covering homotopy property" [H, p. 63] which follows from a slight generalization of the isotopy extension theorem. 
2. The exact sequences. We will use the fibration of $\$ 1$ to analyze the group $D^{\pi}(M)$-see below for a definition. In this section we describe how the addition of a handle affects this group. For the remainder of this section we adopt the following notation (see picture): $H_{k}=D^{k} \times D^{n-k}, \partial^{+} H_{k}=D^{k} \times S^{n-k-1}, a: S^{k-1} \times$ $D^{n-k} \rightarrow \partial M$ is some embedding, $\tilde{M}=M \cup_{a} H_{k}, \bar{i}: H^{k} \rightarrow \tilde{M}$ is inclusion, $i_{1}=$ $\bar{i}\left|\partial^{+} H_{k}, i^{\prime}=\bar{i}\right| 0 \times S^{n-k-1}, i^{\prime \prime}=\bar{i} \mid 0 \times D^{n-k}$. We say that $\tilde{M}$ is obtained from $M$ by addition of a handle of index $k$.

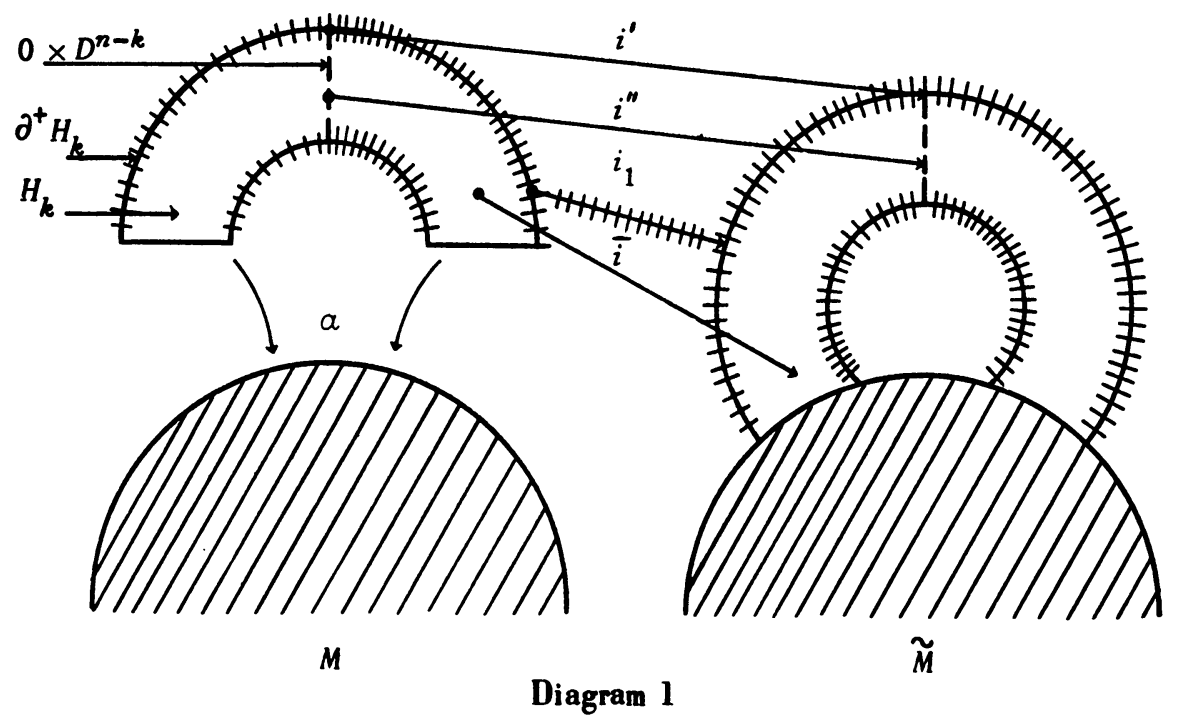

Definition 2.1. $f_{0}, f_{1} \in \mathcal{E}_{i}(N, M)$ are homotopic relative to $i$ if there exists a homotopy $f_{t}$ between them such that $f_{t} \mid N^{\prime}=i$ for all $t$.

$$
\begin{aligned}
& \mathcal{E}_{i}^{\pi}(\tilde{M})=\left\{b \in \mathcal{E}_{i}\left(H_{k}, \widetilde{M}\right) \mid b \text { is homotopic to } \bar{i} \text { relative to } i\right\}, \\
& \mathcal{D}^{\pi}(\tilde{M})=\{f \in \mathscr{D}(\tilde{M}) \mid f \text { is homotopic to } 1 \text { relative to } 1 \mid \partial \tilde{M}\}, \\
& \mathcal{E}_{i}^{\pi}(\tilde{M})=\left\{g \in \mathcal{E}_{i}\left(D^{n-k}, \tilde{M}\right) \mid g \text { is homotopic to } i^{\prime \prime} \text { relative to } i^{\prime}\right\}
\end{aligned}
$$

$\mathscr{D}^{\pi}(M)$ has an analogous definition. We abbreviate $\tilde{D}^{\pi}=\mathscr{D}^{\pi}(\tilde{M}), \mathscr{D}^{\pi}=\mathscr{D}^{\pi}(M)$, $\mathcal{E}_{i}^{\pi}=\mathcal{E}_{i}^{\pi}(\tilde{M}), \mathcal{E}_{i}^{\pi},=\mathcal{E}_{i}^{\pi},(\tilde{M})$ and similarly without the superscript. If $\partial M=\varnothing$ the homotopy conditions on $\mathscr{D}^{\pi}$ read "homotopic to the identity." $\mathscr{D}^{\pi}$ is a group: for if $F, G: M \times I \rightarrow M \times I$ are homotopies from 1 to $f$ and $g$ respectively, then $F \circ G$ and $F^{-1}$ are such homotopies for $f^{\circ} g$ and $f^{-1}$. Here, $F^{-1}$ denotes a homotopy inverse for $F$ on $(M \times I, \partial(M \times I))$ which exists by the following argument: $F$ induces isomorphisms on the homotopy of $M \times l$ and $\partial(M \times l)$ and therefore, by the five lemma, on that of $(M \times l, \partial(M \times l))$. Then by the Whitehead theorem, $F$ is a relative-homotopy equivalence. 
Theorem 2.2. (a) $\mathscr{D} \subset \mathscr{D} \stackrel{R}{\longrightarrow} \mathcal{E}_{i}$ is a fibration, (b) $\mathcal{Q} \subset \mathscr{D}^{\pi} \rightarrow \mathcal{E}_{i}^{\pi}$ is a fibration (tbis defines 2 ), and $\mathfrak{D}^{\pi} \subset \mathcal{Q} .\left(Q=\pi_{0}(\mathcal{P})\right.$.)

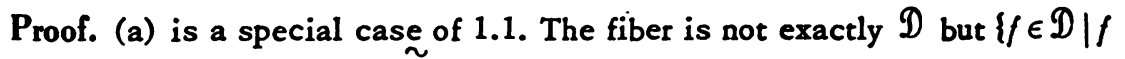
extends to a diffeomorphism of $\tilde{M}\}$. However, these spaces are homotopy equivalent and by recent results of [H \& S] are therefore homeomorphic.

(b) That the fibration of (a) restricts to this fibration follows from the local product structures on base and total space.

In order to understand the step from $D$ to $\tilde{D}$, we consider a portion of the homotopy exact sequence $D \rightarrow \widetilde{D} \rightarrow E$ and look for information about $E$. This is very hard to come by unless the handle has high index (and therefore low dimensional transverse disc). Consideration of (b) is suggested by Browder's homotopy characterization of isotopic embeddings in [B]. The situation is described by the following angle exact diagram (a diagram will be said to be angle exact if it is exact through each nonstraight angle):

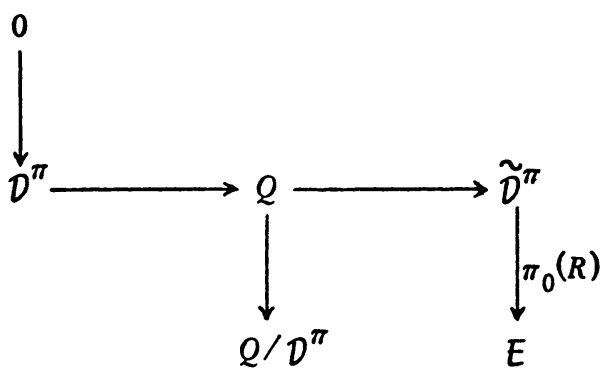

Diagram A

It is the term $E_{i}^{\pi}$ that Browder's result enables us to calculate. In the remainder of $\$ 2$, we discuss primarily the map $\pi_{0}(R)$, whose image can be described in terms of groups of framed knots. $Q / D^{\pi}$ has the following homotopy characterization: Let $\mathcal{H}(M)$ denote the homotopy equivalences of $M$ relative (pointwise) to $\partial M$. Then

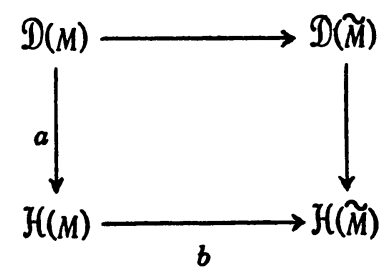

commutes (all maps inclusions) with $\mathcal{Q}=\operatorname{ker}(b \circ a), \mathfrak{D}^{\pi}=\operatorname{ker} a$. Therefore,

$$
2 / D^{\pi}(M) \simeq Q / D^{\pi}(M) \simeq \operatorname{Image}(a) \cap \operatorname{ker}(b) .
$$

This term is finitely generated (a fact that follows from $\$ 3$ ) but can be effectively calculated only if $\widetilde{M}$ retracts to $M$. 
The first step in understanding $\pi_{0}(R)$ is

Theorem 3.4. $\tilde{E}_{i} \stackrel{S}{\rightarrow} \mathcal{E}_{i^{\prime}}$ and $\mathcal{E}_{i}^{\pi} \stackrel{S}{\rightarrow} \mathcal{E}_{i}$, are locally trivial fibrations with fiber $F, \pi_{i}(F) \simeq \pi_{i+n-k}\left(S O_{k}\right)$. [S restricts an embedding of $H_{k}$ to one of $0 \times D^{n-k}$.]

Proof. That $S$ is a fibration in both cases is proven from Palais' lemma [P] using the fact that $\mathscr{E}_{i}$ and $\mathscr{E}_{i^{\prime}}$ are $\mathfrak{D}$ spaces (respectively $\mathscr{G}_{i}^{\pi}$, and $\mathscr{E}_{i}^{\pi}$ are $\mathfrak{D}^{\pi}$ spaces) and $R$ is equivariant with respect to this action. Then $F=\left\{b \in E_{i}|b| 0 \times D^{n-k}=i \mid 0 \times D^{n-k}\right\}$. $F$ can be expressed as $F \simeq C^{\infty}\left(\left(D^{n-k}, S^{n-k-1}\right),(G L(n)\right.$, id $\left.)\right) \times F^{\prime}$ where $F^{\prime}=$ $\left\{b \in F|d b| 0 \times D^{n-k}=d i \mid 0 \times D^{n-k}\right\}$ as follows: for $\alpha \in C^{\circ}\left(\left(D^{n-k}, S^{n-k-1}\right),(G L(n)\right.$, id $\left.)\right)$ let $b_{a}: H_{k} \rightarrow \tilde{M}$ be $b_{a}(x, y)=i(a(y)(x), y)$; then $b$ corresponds to $\left(d b_{0} b_{\left(d b \mid 0 \times D^{n-k}\right)}^{-1}{ }^{\circ}\right)$.

Assertion. $F^{\prime}$ is contractible.

Let $A=\left\{g \in F^{\prime} \mid g\left(D^{k} \times D^{n-k}\right) \subset i\left(D^{k} \times D^{n-k}\right)\right\}$. We first contract $F^{\prime}$ to $A$ and then contract $A$ to a point. Let $\gamma_{t}$ be an isotopy such that $\gamma_{0}=1$ and

$$
i\left(D^{k} \times D^{n-k}\right) \subset U=\gamma_{1}\left(\text { int }\left(D^{k}\right) \times D^{n-k}\right) \text {. }
$$

For $t \in I$, define

$$
N_{t}=\{(u, v)\|\| u \| \leq t \text { or }\|v\| \geq 1-t\} \subset H_{k}
$$

and

$$
m(g)=\max _{t \in I}\left\{t \mid g\left(N_{t}\right) \subset U\right\}
$$

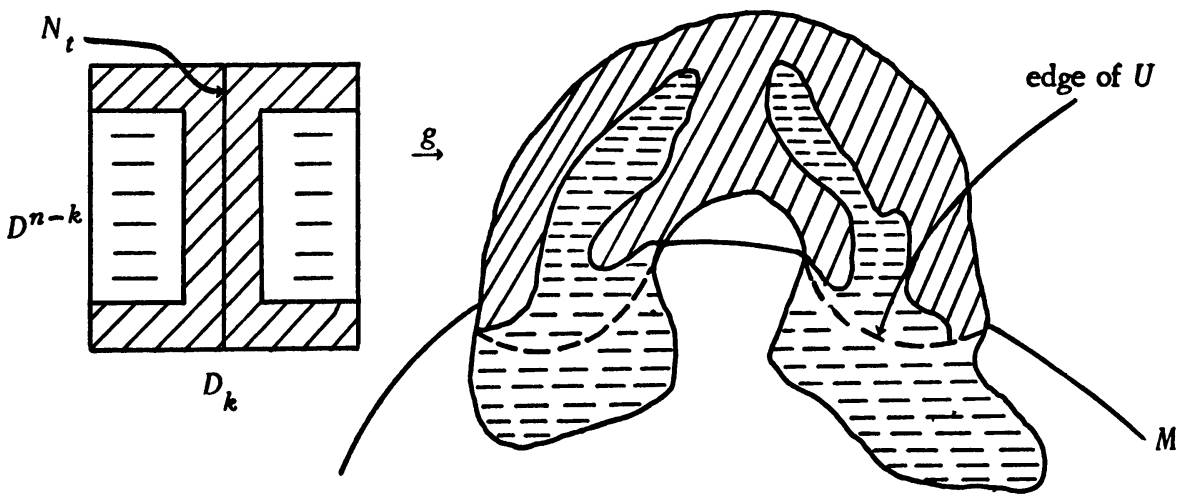

Diagram 2

$m$ is a positive lower semicontinuous function $\left(m^{-1}(b, 1]\right.$ is oben for all $\left.b\right)$ since if $m(g)=q, \tilde{g}\left(N_{q-\epsilon}\right) \subset U$ for $\|g-\tilde{g}\|<\epsilon$. Since $\tilde{E}_{i}$ is paracompact, there is a continuous function $\mathrm{f}(g)$ such that $0<\epsilon(g) \leq m(g)$ for all $g$. (We use the $C^{k}$ topologies for this argument.) 
A contraction of $F^{\prime}$ into $A$ can be described as follows: Let $b(g, v)$ be a continuous furction on $F^{\prime} \times D^{n-k}, C^{\infty}$ in $v$, such that

$$
\begin{array}{ll}
b(g, v)=\epsilon(g) & \text { if }\|y\| \leq 1-\epsilon(g), \\
b(g, v)=1 & \text { if }\|y\|=1,
\end{array}
$$

and $b_{t}(g, y)=(1-t) b(g, v)+t$ be an soitopy between $b_{0}=b$ and $b_{1}(g, y)=1$ (note that $\left.b_{t}(g, v)>0\right)$; then

$$
r_{\imath}(g)(u, v)=g\left(b_{1-t}(g, v) u, v\right)
$$

and $\tilde{C}_{t}(g)=\gamma_{t} \circ C_{1}(g)$. Then $C_{1}(g)\left(H_{k}\right) \subset U$ since $\left(b_{0}(g, v) u, v\right) \in_{g}\left(D^{k} \times D^{n-k}\right)$ and so $\widetilde{C}_{1}(g)\left(H_{k}\right) \subset i\left(H_{k}\right)$.

Finally $A$ can be rontracted to $i$ bv

$$
\begin{aligned}
& d_{t}(g)(u, v)=i r(1-t) i^{-1} g(u,(1-i) v), \quad 0 \leq t<1, \\
& d_{1}(g)(u, v)=i(u, v),
\end{aligned}
$$

where $r(t)(u, v)=(u, v / t)$. The continuity of $d_{t}$ lollows rrom standard techniques, using the fact that $d b=d g$ on $0 \times D^{n-k}$. This completes the proof of the asse. tion. That $F$ has homotopy groups as claimed is now clear:

$$
\begin{aligned}
\pi_{i}(F) & \simeq \pi_{i}\left(C^{\infty}\left(\left(D^{n-k}, S^{n-k-1}\right), G L(n), \text { id }\right)\right) \\
& \simeq \pi_{n-k+1}(G L(n), \text { id }) \simeq \pi_{n-k+1}\left(S O_{n}\right) .
\end{aligned}
$$

Definition 2.5. Let

$$
A=\xi_{i}^{\pi}\left(D^{k} \times D^{n-k}\right), \quad B=\xi_{i}^{\pi}\left(D^{k} \times D^{n \cdot k}\right)
$$

and $C$ be the fiber of the restriction map $B \stackrel{K}{\longrightarrow} A$. (B and $A$ are knots and framed knots respectively in the handlę $H_{k}$.)

For any $M, \widetilde{M}$ and $i$ as in 2.1, there is an inclusion $A \stackrel{a}{\longrightarrow} \mathcal{G}_{i}^{\pi}$ given by $a(f)=\bar{i} \circ f$ which is a fiber map giving the top two squares of the following commutative diagram.

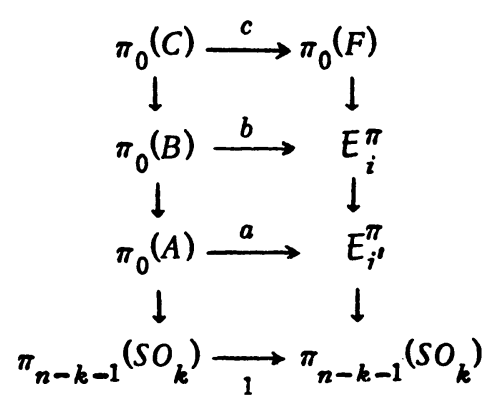

The images of $b$ and $a$ are those knots and framed knots in $\widetilde{M}$ admitting representatives in the handle $H_{k}$. The vertical maps in the bottom square are the 
classifiers of normal bundles (over $\left(D^{n-k}, S^{n-k-1}\right)$ ) and the square commutes since $a$ does not alter normal bundles. The vertical sequences are exact at $\pi_{0}(A)$ and $E_{i}^{\pi}$, since the obstruction to obtaining the desired extensions are exactly the normal bundles.

Lemma 2.6. For $k \geq 3$,

(i) $c$ is an isomorphism,

(ii) $\pi_{0}(B) \simeq F C_{n-k}^{k}$,

(iii) $\pi_{0}(A) \simeq C_{n-k}^{k}$.

(Recall: $C_{n-k}^{k}=E\left(S^{n-k}, S^{n-k} \times D^{k}\right)$ and $F C_{n-k}^{k}=\pi_{0}\left(D_{\text {iff }}\left(S^{n-k} \times D^{k}\right)\right)$ where here Diff means that the boundary is fixed as a set, but not necessarily pointwise.)

Proof. (i) From $2.4, \pi_{0}(F) \simeq \pi_{0}(C) \simeq \pi_{n-k}\left(S O_{k}\right)$ and it is not hard to see that the inclusion induces an isomorphism.

(ii) A map $v: \pi_{0}(A) \rightarrow F C_{n-k}^{k}$ is given as follows: for $f \in A, f: D^{k} \times D^{n-k}$ $\rightarrow D^{k} \times D^{n-k}$

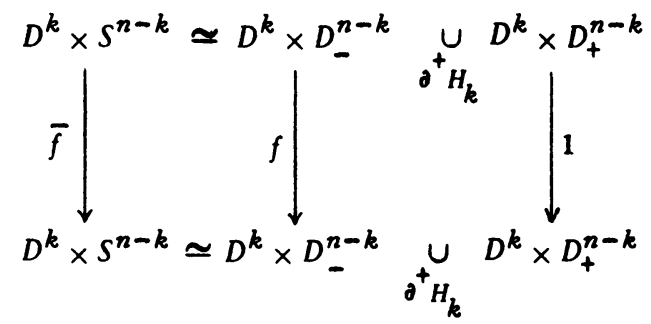

$\bar{f}$ is well defined since $f \mid D^{k} \times S^{n-k-1}=1$; then $v([f])=[\bar{f}]$. Standard techniques (see, e.g., [Ha 1]) show that $v$ is well defined on $\pi_{0}(\mathrm{~A})$ and is an isomorphism.

(iii) Same as (ii) without the framing.

We therefore have a diagram

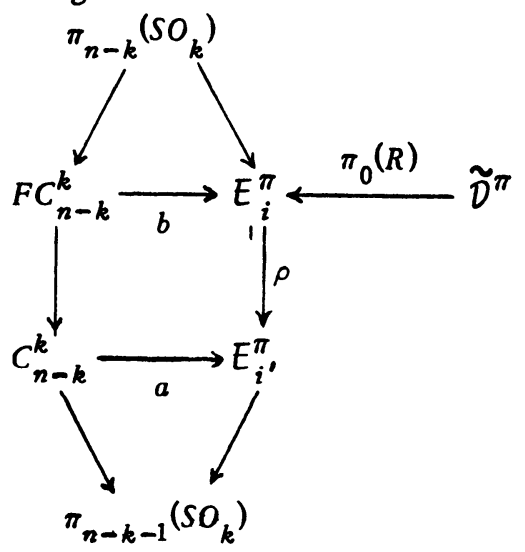

Diagram 3 
(The left vertical sequence is the usual one relating framed and unframed knots.)

It is here that we can use Browder's result.

Theorem. If $M$ is 2-connected, $f: N^{n-k} \rightarrow M, k>2, \pi_{1}(N)=0$ and $d: M \rightarrow M$ is a diffeomorphism bomotopic to 1, then $d \circ f$ and $f$ are isotopic modulo local knotting: i.e., for some knotted $D^{n-k}$ in $D^{n}$ and $f^{\prime}$ differing from $f$ by exactly that knot near some point, $f^{\prime}$ is isotopic to $d \circ \%$.

Proof. The data for Browder's theorem are:

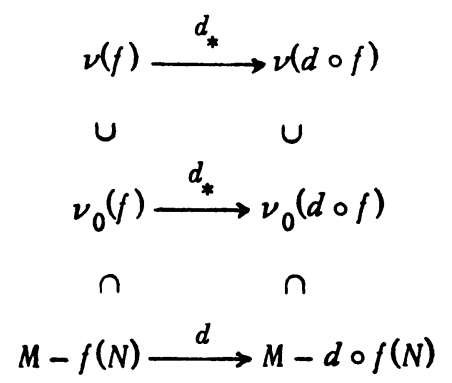

which fit together to give $d: M \rightarrow M$. ( $\nu$ and $\nu_{0}$ denote normal disc and sphere bundles respectively.) As $d$ is assumed to be homotopic to 1 and the necessary homology conditions follow from the connectivity, the result follows.

Theorem 2.7. In Diagram 3

$$
\text { Image }\left(\pi_{0}(R)\right) \subset \text { Image }(B)
$$

if $\pi_{1}(M)=\pi_{1}(\partial M)=\pi_{2}(M)=0$.

Proof. It suffices to show that $a$ is onto Image $\left(\rho \circ \pi_{0}(R)\right)$ : a diagram chasing argument then shows that $b$ is onto Image $\left(\pi_{0}(R)\right)$. Notice that in the metastable range $(k \geq(n / 3)+1)$ there is no problem-all homotopies can be replaced by isotopies and $E_{i}^{\pi}=0$. In general, though, we need Browder's result, whose terminology we now adopt. The group $J=J\left(D^{n-k}, S^{n-k-1}\right.$ ) (we are in the relative. case) is $C_{n-k}^{k}$ and the statement that two embeddings in $\mathcal{E}_{i}^{\pi}$, are isotopic (same as pseudo-isotopic by [C] since $\tilde{M}$ is simply connected) under the action of $J$ is equivalent to saying that they differ by an element of Image(a). But the above result shows that any element in $\mathbb{E}_{i}^{\pi}$, differs from inclusion by local knotting-thus every element is in Image $(a)$.

For a description of $\Pi_{0}\left(\tilde{D}^{\pi}\right) / \Pi_{0}\left(D^{\pi}\right)$, then, we are left with the problem of understanding $\Pi_{0}(Q) / \Pi_{0}\left(D^{\pi}\right)$. If $\tilde{M}$ retracts to $M$, this can be done. See [T].

3. Finite generation and commutativity. The inductive procedure of $\$ 2$ can be applied to show that $D^{\pi}(M)$ is finitely generated (if $\pi_{1}(M)=0$ ). This and more can be shown in more generality by relating $D^{\pi}(M)$ to an exact sequence 
from the theory of surgery. (The author is indebted to the reviewer for suggesting this approach.)

Definition. $b \mathcal{D}(M)=\{H: M \times I \rightarrow M \times I|H| M \times 0 \cup \partial M \times I=1, H \mid M \times I \in \mathcal{D}(M)$ and $H$ is a simple homotopy equivalence $\}$ and $b D(M)=\pi_{0}(b D(M))$. Then $b D(M)$ is a group under composition and the restriction $H \rightarrow H \mid M \times 1$ defines an epimorphism

$$
\phi: b D(M) \rightarrow D^{\pi}(M)
$$

where $D^{\pi}(M)$ is defined in the nonsimply connected case with homotopies that are simple homotopy equivalences. (If one does not make such a restriction, the results are complicated.in a rather obvious way by obstructions in $W b\left(\pi_{1}(M)\right)$.) Let $\mathcal{P} \subset b D(M)$ denote the set of diffeomorphisms (i.e. pseudo-isotopies) and $P$ be the image of $\pi_{0}(\mathcal{P}) \rightarrow b D(M)$. Then $\phi(P)$ is subgroup of $D^{\pi}(M)$ consisting of those diffeomorphisms pseudo-isotopic to 1 , so $D^{\pi}(M) / \phi(P)$ is a subgroup of the "concordance homotopy group" $\pi_{0}(\mathrm{Diff}: M)$ in the notation of [ABK]. Other notation in this section will be as in [W].

Theorem 3.1 There are exact sequences

(a) $0 \rightarrow P \rightarrow b D(M) \stackrel{a}{\rightarrow} \delta[M \times I] \rightarrow 0$.

(b) $L_{n+1}\left(\pi_{1}(M)\right) \rightarrow \delta[M \times I] \rightarrow[\Sigma M, G / 0] \rightarrow L_{n}\left(\pi_{1}(M)\right)$.

The corollaries exploit the fact that $[\Sigma M, G / 0]$ is a finitely generated abelian group since $\pi *(G / 0)$ is of finite type, $G / 0$ is an $H$-space and $M$ is compact.

Corollary 1. If $L_{n+1}\left(\pi_{1}(M)\right)$ is finitely generated (e.g. if $\pi_{1}(M)$ is finite or finitely generated abelian) then $D^{\pi}(M) / \phi(P)$ is finitely generated.

Corollary 2. If $\pi_{1}(M)=0$, then $D^{\pi}(M) / b P_{n+2}$ is finitely generated abelian where $b P_{n+2} \subset \Gamma_{n+1} \rightarrow D^{\pi}(M)$. Thus, if $n$ is odd, $D^{\pi}(M)$ is finitely generated abelian.

Proofs. The corollaries follow easily from the theorem, using the facts that for $\pi_{1}(M)=0, P=0 ; b P_{n+2}=\partial L_{n+2}(0)$; and the diagram

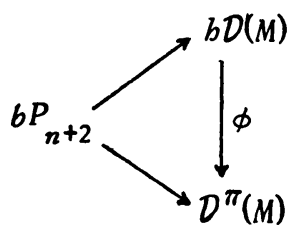

commutes. Sequence (b) is a central result of surgery theory; see, e.g., [W, p. 112].

The map $a$ of a sequence is onto by the following argument: any smoothing in $S[M \times I]$ is represented by a simple homotopy equivalence $L \stackrel{f}{\longrightarrow} M \times I$ and, by the $s$-cobordism theorem, $L$ must be diffeomorphic $M \times I$; composing with this 
diffeomorphism gives an equivalent smoothing $M \times I \stackrel{f^{\prime}}{\longrightarrow} M \times I$; further composition with $\left(f^{\prime} \mid M \times 0\right)^{-1} \times 1$ gives a representative whose class is clearly in the image of $a$. The kernel of $a$ is $P$ by definition of $\delta[M \times I]$. The only point that requires checking is that $a$ is a homomorphism. If $H_{1}, H_{2} \in b D(M)$, then $H_{1}$ is equivalent to $H_{1}^{\prime}$ concentrated on $M \times[1 / 2,1]$ (and 1 on $M \times[0,1 / 2]$ ) and $H_{2}$ to an $H_{2}^{\prime}$ concentrated on $M \times[0,1 / 2]$ (and $\left(H_{2}^{\prime} \mid M \times 1\right) \times 1$ on $M \times[1 / 2,1]$ )-then $H_{2}^{\prime} \circ H_{2}^{\prime}$ amounts to "stacking" the smoothings $H_{1}$ and $H_{2}$, which is the group operation in $S[M \times I]$. (Remark. The existence of inverses for this operation is not obviousit follows from either sequence (a) or (b).)

\section{REFERENCES}

[ABK] P. L. Antonelli, D. Burghelea and P. J. Kahn, The concordance homotopy groups of geometric automorphism groups, Lecture Notes in Math., vol. 215, Springer-Verlag, Berlin and New York, 1971.

[B] W. Browder, Embedding 1-connected manifolds, Bull. Amer. Math. Soc. 72 (1966), 225-231. MR 32 \#6467.

[C] J. Cerf, The pseudo isotopy theorem for simply connected differential manifolds, Manifolds-Amsterdam 1970 (Proc. Nuffic Summer School), Lecture Notes in Math., vol. 197, Springer, Berlin, 1971, pp. 76-82. MR 44 \#7585.

[Ha 1] A. Haefliger, Differential embeddings of $S^{n}$ in $S^{n+q}$ for $q>2$, Ann. of Math. (2) 83 (1966), 402-436. MR 34 \# 2024.

[H\&S] D. W. Henderson and R. Schori, Topological classification of infinite dimensional manifolds by homotopy type, Bull. Amer. Math. Soc. 76 (1970), 121-124. MR 40 \# 4976.

[H] S. T. Hu, Homotopy theory, Pure and Appl. Math., vol. 8, Academic Press, New York, 1959. MR 21 \#5186.

[P] R. Palais, Local triviality of the restriction map for embeddings, Comment. Math. Helv. 34 (1960), 305-312. MR 23 \# A666.

[S] D. Sullivan, Triangulating homotopy equivalences, $\mathrm{Ph}$. D. thesis, Princeton Univo, Princeton, N. J., 1966.

[T] E. C. Turner, Some finite diffeomorphism groups, Illinois J. Math. (to appear).

[w] C. T. C. Wall, Surgery on compact manifolds, Academic Press, New York, 1971.

DEPARTMENT OF MATHEMATICS, STATE UNIVERSITY OF NEW YORK AT ALBANY, ALBANY, NEW YORK 12222 